\title{
DESARROLLO DE LA COMPETENCIA GLOBAL EN LA FORMACIÓN DEL MAESTRO. EL CASO DE LA ASIGNATURA PRACTICUM
}

\section{DEVELOPMENT OF GLOBAL COMPETENCE AMONG STUDENTS OF EDUCATION THROUGH THE PRACTICUM SUBJECT}

http://dx.doi.org/10.15304/ie.28.5361

\author{
Inmaculada Gómez-Jarabo \\ Universidad Complutense de Madrid \\ inma.gomez@edu.ucm.es
}

M a José Cornejo Sosa

Universidad Autónoma de Madrid

mjcornejososa@gmail.com

\section{RESUMEN}

El hecho de que las aulas de infantil y primaria sean cada vez más multiculturales hace necesario que los futuros maestros posean un grado de dominio alto en competencia intercultural. Así lo señala la OCDE, a través de la evaluación de PISA, al incluir para 2018 la evaluación de la competencia global definida como "capacidad de analizar asuntos globales e interculturales, valorar distintas perspectivas desde el respeto por los derechos humanos, para interrelacionarse con personas de diferentes culturas emprender acciones por el bien común y por el desarrollo sostenible" (OCDE, 2016, p. 4). Sin embargo, si observamos los programas de los actuales Grados, la presencia de asignaturas y orientaciones que faciliten el conocimiento de asuntos globales, las destrezas de pensamiento crítico y las actitudes de apertura y empatía hacia puntos de vista diferentes al propio, no es suficiente. En este sentido, el objetivo de este artículo es doble. En primer lugar, llamar la atención sobre la escasa preparación del estudiante universitario, en concreto de los Grados de Educación Infantil y Primaria, como responsable de desarrollar esta competencia en las siguientes generaciones y, en segundo lugar, proponer la asignatura de Practicum como medio propicio para desarrollar una pedagogía que familiarice a los chicos con diferentes contextos culturales.

Palabras clave: competencia global, formación docente, evaluación, Practicum, intercultura.

\begin{abstract}
Increasingly multicultural infant and primary classrooms require future teachers to possess a high domain of intercultural competence as indicated by the OECD through the evaluation of PISA which includes for this 2018 the evaluation of global competence defined as the "ability to analyze global and intercultural issues, assess different perspectives reflecting the respect for human rights, to interrelate
\end{abstract}


with people from different cultures and undertake actions for a common good and a sustainable development" (OECD, 2016, p. 4). However, if we look at the programs of the current Degrees, the presence of subjects and orientations which facilitate the knowledge and understanding of global issues, the critical thinking skills and the attitudes of openness and empathy towards points of view different from one's own, is not enough. Thus, the objective of this article is twofold. First, call attention to the poor preparation of the university student, specifically the Degrees of Infant and Primary Education, as the responsible for developing this competence in the following generations and, secondly, to propose the subject of Practicum as a favorable medium to develop a pedagogy that familiarizes children with different cultural contexts.

Keywords: global competence, education degree, assessment, Practicum, interculture.

\section{INTRODUCCIÓN}

A partir de las directrices de Bolonia, España, junto al resto de Europa, está inmersa en un proceso de convergencia para responder más eficazmente a las necesidades formativas del estudiante universitario y mejorar su inserción laboral en una sociedad cada vez más compleja. Complejidad que deviene, en parte, de la diversidad cultural que conforman instituciones, equipos humanos, interacciones comerciales, etc., propias de un mundo globalizado.

La globalización conlleva mayor apertura y conectividad, pero también cierta desigualdad y riesgo de radicalismo (Ramos y Schleicher, 2016), de ahí que la OCDE (2016) apunte a la necesidad perentoria de desarrollar la competencia global en la formación integral del estudiante del siglo XXI. Se trata de desarrollar en el alumno habilidades de análisis y comprensión de problemas de ámbito global y competencias sociales que incluyan habilidades para interrelacionarse con personas de diferentes culturas y valores de tolerancia a diferentes perspectivas desde el criterio, sentido de pertenencia, empatía, etc., que son fundamentales para superar la propia "nacionalidad cultural" y avanzar hacia una sociedad global interdependiente e inclusiva.

Sin embargo, basta echar una mirada a los programas de los actuales Grados para cuestionarse si la presencia de asignaturas y de orientaciones que desarrollen esa competencia global es suficiente, así lo señalan numerosas investigaciones que, tomando como referencia diferentes zonas y regiones de España y con uso de diferentes metodologías, vienen concluyendo desde hace años que el profesorado no se prepara para dar respuesta a la realidad multicultural de los centros educativos (Aguado Odina, 2006; Calvo Buezas, 2003; Domínguez Garrido, 2006; García López, 2002; Gómez Barreto, Medina Revilla y Gil Madrona, 2011; Gómez-Jarabo, 2015: Leiva Olivencia, 2012; LópezReillo, 2006; Mingote y Requena, 2008; Palomero, 2006; Rodríguez Izquierdo, 2008). En este sentido, el objetivo de este artículo es doble. En primer lugar, llamar la atención sobre la escasa preparación del estudiante universitario, en concreto de los Grados que habilitan para la profesión de maestro, como figura responsable de desarrollar esta competencia en las siguientes generaciones y, en segundo lugar, proponer la asignatura del Practicum, como asignatura propicia para este desarrollo, dado el tipo de conocimiento y el tipo de aprendizaje que propicia (Méndez, 2012).

Comenzamos describiendo el actual concepto de interculturalidad y sus implicaciones desde la perspectiva educativa, dentro de lo que la OCDE denomina competencia global; en relación con ello, analizamos la formación que los estudiantes de esos Grados reciben sobre interculturalidad 
para relacionar esa formación con los periodos en los que realizan prácticas actualmente. A partir de ahí, realizamos una propuesta para mejorar la preparación del estudiante antes de las prácticas, respecto a esta competencia global; para ello, utilizaremos el modelo didáctico de dimensiones culturales propuesto por Cornejo, Gómez-Jarabo y Jiménez (2017), desde el que se asume que la mayoría de los valores, actitudes y comportamientos son prácticas culturales que se reflejan en la interacción comunicativa.

\section{EL PENSAMIENTO INTERCULTURAL EN LA COMPETENCIA GLOBAL}

En 2013, la OCDE inicia el proyecto "The Future of Education and Skills: an OCDE Education 2030 Framework", con el objetivo de definir las necesidades de aprendizaje y las competencias básicas necesarias para las próximas décadas. No hay que olvidar que es una Organización para la Cooperación y para el Desarrollo Económico de los países, que contempla la educación como medio para lograr este desarrollo. Quizá por este matiz económico, posee una visión pragmática y anticipadora del tipo de sociedad que tendremos y, en función de ello, realiza una llamada de atención a los gobiernos de los distintos países sobre las necesidades educativas que genera. De alguna manera, "obliga" a llevarlas a cabo desde la educación primaria mediante un instrumento ya sobradamente conocido, PISA (Program for International Student Assessment).

Una de las necesidades educativas percibidas por la OCDE dentro del proyecto marco que señalamos en el párrafo anterior, es la necesidad de tratar las demandas y los desafíos que devienen de la inevitable globalización, con dos claras implicaciones educativas. La primera, es que ya desde la etapa primaria y de forma transversal, no podemos circunscribirnos a tratar temas y contenidos propios de nuestro ámbito geográfico. Temas como el calentamiento global, la sostenibilidad, los avances tecnológicos, etc., deben tratarse desde distintas perspectivas geográficas, si queremos avanzar hacia una sociedad global interdependiente. La segunda implicación es el hecho de que las futuras generaciones trabajarán en contextos con personas de diferentes disciplinas, culturas y creencias que poseen diferentes visiones ante un mismo problema. La constatación de este último hecho puede presentarse como un problema, si no actuamos, o bien como una oportunidad para desarrollar actitudes de apertura y de flexibilidad que permitan identificar rasgos culturales propios, prejuicios y, sobre todo, que permitan reconocer que nuestra comprensión del mundo es inevitablemente parcial.

Ahora bien, ¿Cómo se instrumentalizan esas constataciones teóricas? El trabajo de la OCDE ofrece una definición formal de competencia global, que es un punto de partida para dar respuesta a esta cuestión: "capacidad de analizar asuntos globales e interculturales, valorar distintas perspectivas desde el respeto por los derechos humanos, para interrelacionarse con personas de diferentes culturas, emprender acciones por el bien común y por el desarrollo sostenible" (OCDE, 2016, p. 4). Si observamos detenidamente esta definición, podemos identificar tres frentes desde los que podemos desarrollar la competencia global en el aula.

El primero de ellos es la constatación de que todos somos extranjeros/inmigrantes en el momento en el que traspasamos la frontera geográfica. Por ello, es necesario trabajar no solo en el sentido de enseñar nuestra cultura al otro, sino también en sentido inverso, de que el otro nos 
enseñe su cultura. Esta primera asunción llevada al aula, supone adoptar el modelo de sensibilidad intercultural de Bennett (1993), y trabajar no solo desde los clásicos estereotipos tales como "los alemanes son cuadriculados", "España: fiesta y toros", que proporcionan una imagen mental muy simplificada, casi siempre negativa, y sesgan las expectativas sobre lo que se puede esperar de un miembro de una determinada cultura al generalizarlo a todos los miembros de la misma. Tampoco se trata de acercarnos a la otra cultura desde el etnocentrismo o desde un relativismo cultural, que proponen un conocimiento y análisis de otras culturas, pero sin encuentro real. Se trata de asegurar un entendimiento compartido por personas de diferentes identidades sociales y de desarrollar la capacidad de interactuar con las personas como seres humanos complejos con múltiples identidades y al tiempo con su propia individualidad (Byram, Gribkova y Starkey, 2002; Deardorff, 2006 y Kazepides, 2012) que, partiendo del reconocimiento mutuo de valores y formas de vida, propugne:

a) El conocimiento de procesos sociales y personales.

b) Las actitudes de curiosidad y apertura, disposición a suspender la incredulidad sobre otras culturas, así como la creencia acerca de la voluntad propia de relativizar sus propios valores, creencias y comportamientos, sin asumir que son los únicos posibles y naturalmente correctos.

c) Las habilidades de interpretar una situación de interactuación con otra cultura, explicarla y relacionarla con eventos propios, así como la capacidad de adquirir conocimiento de la misma.

Es en la capacidad de cambiar el marco propio de referencia donde reside el núcleo de la competencia intercultural (Deardorff, 2006).

El segundo frente para desarrollar el pensamiento intercultural del alumno está en estrecha relación con el anterior. Se trata de trabajar el binomio lengua-cultura como base fundamental de interacción. Si bien las investigaciones sobre intercultura tienen su origen en la enseñanza tradicional de la cultura, para la cual la lengua y la cultura son realidades disociables, ya desde los años 80 se apuesta por la existencia de un fuerte vínculo entre ambas. El dominio de la lengua no es garantía absoluta de integración. El 36,4 \% de los alumnos inmigrados en España provienen de América del Sur y no están exentos de exclusión social ${ }^{1}$. Esta asunción conlleva trabajar la capacidad del alumno para reconocer la validez de otras formas de expresar la experiencia que afecta a cómo nos expresamos, qué fórmulas se utilizan por ejemplo para el acto de pedir u ofrecer y cómo se usa la cortesía verbal para salvaguardar la imagen del otro. Se trata de introducir el ingrediente pragmático de la lengua como parte del pensamiento intercultural.

El tercer frente de trabajo es la necesidad de cubrir el vacío formativo de los futuros maestros en la competencia global, que desarrollamos a continuación.

Fuente: Informe de la Asamblea General de las Naciones Unidas, 2016. Migración internacional y desarrollo. 


\section{LA FORMACIÓN EN INTERCULTURALIDAD EN LOS GRADOS DE EDUCACIÓN}

La inclusión de la competencia global como tema en la próxima encuesta internacional de enseñanza y aprendizaje de la OCDE (TALIS), señala la necesidad de analizar la efectividad de la preparación de los maestros en la competencia global. Sin embargo, los profesionales educativos ya en ejercicio siguen encontrando dificultades para gestionar eficazmente la diversidad cultural; es más, la perciben como una dificultad que incapacita para seguir el ritmo de la actividad escolar normal (Díaz-Aguado 2002) y la abordan, normalmente, derivando a los alumnos inmigrados hacia otros profesores fuera del aula ordinaria, los profesores de apoyo, que a su vez se centran en enseñar el idioma que les permita seguir el ritmo. Por ello, el profesorado requiere de formación específica para atender a todo el alumnado, reconducir los conflictos habituales en la convivencia y caminar hacia una sociedad global más democrática y pluralista. A pesar de ello, en ocasiones el profesorado en ejercicio no recibe la formación que le permita hacer frente a todas sus responsabilidades, lo que le lleva a actuar por "sentido común" o repetir las estrategias que empleaban con ellos sus docentes, sean estas adecuadas o no. En este sentido, nos surge una pregunta: ¿Se está preparando adecuadamente a los estudiantes de los Grados de Educación (Maestro en Educación Infantil, Maestro en Educación Primaria, Pedagogía y Educación Social), para trabajar con la diversidad cultural que encontrarán en las aulas?

García López (2002) mostró en una investigación cómo de un total de 155 planes de estudio de 42 universidades españolas únicamente existían 62 asignaturas relacionadas con la "educación intercultural y multicultural" y con la "diversidad sociocultural”. Podría parecer que 62 es una buena cifra, pero el problema es que únicamente 8 de ellas eran de carácter obligatorio. Las 54 asignaturas restantes tenían carácter optativo, lo que no asegura la formación de todos y todas. En la misma línea, Mingote y Requena (2008, p. 214) comentan:

En las Facultades de Pedagogía o Escuelas de Magisterio no se enseña a los profesores a ser líderes democráticos y competentes que transmitan expectativas positivas, precisas y flexibles, individualizando la enseñanza para que cada alumno participe, compensando las diferencias existentes entre ellos.

Quizá podría pensarse que la situación hoy en día es diferente, pues los trabajos de García López y de Mingote y Requena ya tienen algunos años. Sin embargo, la investigación de GómezJarabo (2015), muestra que las asignaturas relacionadas con la interculturalidad no abundan en las titulaciones de educación (Grado de Educación Social, Grado de Pedagogía, Grado de Maestro en Educación Infantil y Grado de Maestro en Educación Primaria). Concretamente, en la Facultad de Educación de la Universidad Complutense de Madrid se imparten dos optativas relacionadas con la interculturalidad en el Grado que habilita para ejercer la profesión de Maestro en Educación Infantil: "Aspectos didácticos de la escuela inclusiva" y "La dimensión intercultural en el curriculum”, en segundo y cuarto curso respectivamente; y una en el Grado que habilita para ejercer la profesión de Maestro en Educación Primaria: "Aspectos didácticos de la escuela inclusiva", en segundo curso. Por tanto, el problema persiste; en la mayoría de los casos, las asignaturas no tienen carácter obligatorio, por lo que una buena parte de los futuros y las futuras profesionales de la educación no reciben una formación que debería ser obligatoria para ellos y ellas por la diversidad actual existente en los centros educativos. 
La investigación de Gómez-Jarabo (2015) complementó el análisis documental con la realización de cuestionarios, entrevistas y grupos de discusión a estudiantes y egresados de otras universidades que, en su mayoría, afirmaron no haber recibido formación para la mejora de la convivencia entre culturas. El problema no acaba ahí, pues al preguntar a los encuestados cómo consideraban que había sido su formación, un 52,2\% de personas del Grado que habilita para ejercer la profesión de Maestro en Educación Infantil y un 85,7\% del que habilita para ejercer en Educación Primaria consideraban que había sido "mala". Las personas que no se mostraban satisfechas con la formación recibida, argumentaban de forma mayoritaria que no se había profundizado en aspectos esenciales, que no se habían proporcionado pautas reales para la intervención y para su tratamiento en los programas educativos y que la formación había sido escasa y demasiado teórica. Ante ese panorama, no extraña que al preguntar a los encuestados si se sentían capacitados para trabajar en la mejora de la convivencia entre culturas, la opción mayoritaria de respuestas se encontrara en "no, apenas", elegida por un 52\% de personas del Grado que habilita para ejercer en Educación Infantil y un $75 \%$ del Grado que habilita para ejercer en Educación Primaria.

Sin embargo, la investigación también mostraba un aspecto muy positivo; muchas de las personas participantes mencionaban la importancia de esta temática para los y las profesionales de la educación, no solo de cara a satisfacer las necesidades del alumnado lo mejor posible y a fomentar la convivencia saludable en sus aulas, sino también a eliminar determinados prejuicios y estereotipos de su persona. Muchos participantes consideraban que se trata de una temática realmente necesaria debido a los cambios sociales que vivimos. A continuación, se muestran algunos de los comentarios que realizaron los participantes en la investigación:

- "Teniendo en cuenta que vivimos en un mundo en el que la convivencia entre personas de etnias muy diversas es un hecho más que frecuente, considero que los programas de formación de profesionales de la educación deberían incluir temas en este sentido ya que no solo aportaría aprendizajes positivos para nosotros como ciudadanos, sino que mejoraría nuestra competencia profesional entendiendo que seremos nosotros quien formará al rango más joven de la sociedad. Desde la educación se alcanzan los grandes avances sociales".

- "En mi opinión, la escuela (y la formación inicial del profesorado) tiene identificados algunos de los "problemas" que se generan de la interculturalidad (incorporación tardía, conflictos frecuentes como la falta de adaptación o el rechazo, ...), pero no se profundiza en las estrategias para afrontar estas realidades, ni se dan a conocer experiencias que ponen en valor la educación intercultural de una forma real (por ejemplo, experiencias de comunidades de aprendizaje o proyectos concretos desarrollados en centros concretos). Esto facilitaría, a mi entender, la buena praxis de los futuros docentes".

- "Creo que la interculturalidad debería tratarse mucho más en esta formación inicial, ya que cuando sales al mundo laboral encuentras gente de otras culturas en las aulas, en las calles, compañeros de trabajo, etc. Y mucha gente, a pesar de haber estudiado, vive de estereotipos y prejuicios acerca de las personas que son de otra cultura distinta a la nuestra". 
- "Debería ser prescriptiva pues la diversidad de la sociedad actual lo impone". (GómezJarabo, 2015, p. 281)

Siguiendo la línea apuntada por los participantes en la anterior investigación, consideramos que el desarrollo de la competencia global es fundamental para todos los docentes. Las asignaturas optativas no permiten una formación universal, sino solo la de las personas que están más sensibilizadas en el tema o las que tienen horarios más compatibles con el de impartición de la asignatura. Asimismo, se debería velar por el incremento del número de asignaturas ofertadas al respecto.

\section{EL PRACTICUM EN LOS GRADOS DE EDUCACIÓN INFANTIL Y PRIMARIA}

La asignatura denominada "Practicum" tiene como finalidad impulsar una formación integral al complementar la formación teórica con la formación práctica, a través del contacto con escenarios reales y con las labores que los estudiantes desempeñarán una vez graduados. Así, lo indica el Real Decreto 1393/2007, de 29 de octubre, de Ordenación de las enseñanzas universitarias oficiales, en relación con las prácticas: “formación que proporciona al alumnado un conocimiento más profundo acerca de las competencias que necesitarán en el futuro".

Las Órdenes ECI/3854/2007 y ECI/3857/2007, del Ministerio de Educación y Ciencia, de 27 de diciembre, que establecen los requisitos de verificación de los títulos universitarios ofíciales de Maestro en Educación Infantil y en Educación Primaria disponen una fase de Practicum con carácter presencial en centros educativos, mediante convenios entre las Administraciones Educativas y las universidades; la planificación del Practicum es competencia de las universidades a las que pertenecen las facultades y escuelas donde se cursen dichos estudios. De esa forma, se pretende la iniciación de los estudiantes en la práctica docente directa, además de conocer los aspectos organizativos, pedagógicos y de funcionamiento de los centros bajo la tutela de profesores y maestros en ejercicio con características y preparación adecuadas. Se trata, así pues, de un tipo de conocimiento diferente al académico, un conocimiento situado propio de los entornos laborales (Méndez, 2012), caracterizado por procesos de interacción social que propician comunidades de aprendizaje de identidad común y que conlleva la activación de estrategias de resolución de problemas en situaciones auténticas. Los beneficios de cursar el Practicum son incuestionables, igual que los inconvenientes de no cursarlo. Sánchez Delgado (2009, p. 4) afirma:

Comenzar la práctica profesional con plena responsabilidad siendo inexperto puede conducir a experiencias que condicionarán el resto de la vida profesional hasta el punto de llevar a algunos a cambiar de profesión o a desarrollarla de modo deficiente en perjuicio propio y de los que deben recibir la atención profesional (...) En educación no son una rara excepción los profesionales que abandonan para siempre en los primeros meses o años de ejercicio. ¿Tendrá algo que ver la realización o no, y las características de la realización del Practicum? Los profesionales de la educación arrojan uno de los más altos porcentajes de consultas psiquiátricas. ¿Algunos de esos profesionales no habrán llegado a esa situación por no haber dispuesto de un Practicum en el que aprender a enfrentar adecuadamente situaciones tan complejas como las que se presentan diariamente en los centros educativos sin sentirse plenamente responsables y disponiendo de un tutor capaz de orientarle adecuadamente? 
Quizá sea la posibilidad que brinda el Practicum de acercarse a contextos reales de intervención la que haga que sea una asignatura tan valorada por los estudiantes y tan motivadora para ellos. En la investigación realizada por Gómez-Jarabo (2015), se solicitó a profesores en activo que describieran las actividades formativas que mejor les habían capacitado para mejorar la convivencia o resolver conflictos. La gran mayoría de las respuestas se centraron en describir formaciones prácticas y se encontró en un lugar muy destacado el Practicum y todo lo relacionado con la actuación en contextos reales.

Aunque la formación práctica es diferente en función de las titulaciones y de los centros de estudio, en el caso de la formación de maestros lo habitual es la distribución de los periodos de prácticas en tres momentos (Practicum I, II y III) y con diferentes objetivos y responsabilidades. En los Grados de Educación Infantil y Primaria, las actividades formativas durante el Practicum están diseñadas para el desarrollo de competencias en las áreas profesional, pedagógica, disciplinar, lingüística e intercultural. En relación con las competencias del área intercultural, los estudiantes deben analizar la respuesta educativa a la diversidad cultural, contrastarla con la formación adquirida en la Universidad y ser capaces de diseñar intervenciones didácticas respetuosas y consecuentes con la diversidad presente en el aula. Dado que no pueden plantearse intervenciones sobre algo desconocido, es preciso que los estudiantes se formen en interculturalidad para poder diseñar acciones educativas coherentes con la misma. Sin embargo, como se comentaba en el apartado anterior, existe una tímida presencia de asignaturas y orientaciones relacionadas con la interculturalidad.

El Practicum puede ser el momento idóneo para conocer mejor la realidad y para aprender todas aquellas cosas que no ha sido posible aprender en el aula, desterrar mitos, falsas creencias y esquemas no válidos, poner en práctica aquellos aspectos que se han aprendido de una forma teórica y conocer otras realidades. No obstante, las prácticas no siempre pueden ayudar a los estudiantes en ese amplio cometido, pues durante el periodo de prácticas pueden no surgir determinadas situaciones, pueden no estar presentes estudiantes con problemáticas concretas o, lo que es peor, pueden observarse formas de intervención no apropiadas o desfasadas, lo que puede conllevar que prejuicios, estereotipos y falsas creencias, ya interiorizadas previamente, se incrementen. En ese sentido, coincidimos con Sánchez Delgado (2009, p.7) cuando afirma:

Debería ponerse especial cuidado para seleccionar determinados centros en función de su capacidad para contribuir en la formación práctica de los futuros profesores. No se trata de que los alumnos del Practicum vivan situaciones de un sólo tipo, sino de que tengan la posibilidad de vivir situaciones didácticas diversas y comprobar la potencia explicativa de las teorías para el análisis de esas situaciones prácticas.

En la misma línea de pensamiento se encuentran Rodríguez Marcos, González Aguado, Egea Reche y Gutiérrez Ruiz (2004), cuando sugieren que habría que cuidar las aulas en las que los estudiantes realizan el Practicum para el logro de la interculturalidad en el trabajo con realidades culturalmente diversas. Estos autores realizaron un estudio con 25 estudiantes de Magisterio (de Educación Infantil y Primaria) para conocer sus creencias respecto a la interculturalidad y la escolarización de gitanos e hijos de inmigrantes y encontraron que: 
- Un $24 \%$ de estudiantes entendía la diversidad cultural como asimilación a la cultura mayoritaria.

- La mayoría de estudiantes consideraba que no había recibido formación adecuada en relación a la interculturalidad ( $84 \%$ respecto a los gitanos y $80 \%$ respecto a los inmigrantes en general). También manifestaron un escaso conocimiento de las culturas minoritarias presentes en nuestro país.

- Un $20 \%$ de estudiantes se sintieron inquietos por no saber cómo actuar con el alumnado gitano y un $16 \%$ con respecto al alumnado marroquí. Además, el no conocer sus costumbres, llevó a un $4 \%$ a rechazar a los latinoamericanos, a un $8 \%$ a los gitanos y a un $16 \%$ a los marroquíes.

- Aunque la mayoría de los estudiantes encuestados manifestaban actitudes de aceptación hacia el colectivo gitano y los hijos e hijas de inmigrantes marroquíes y latinoamericanos, al preguntarles cómo los imaginaban, la gran mayoría se refirió a ellos como problemáticos o con algún tipo de problema ( $92 \%$ en el caso de los marroquíes, $40 \%$ en el caso de los latinoamericanos).

Ante tales datos, nos surgen algunas inquietudes: ¿Cómo actuarán esos futuros profesionales en un futuro si no reciben una mayor formación e información?, ¿podrán detectar situaciones discriminatorias?, ¿qué ocurre si en sus periodos de prácticas coinciden con profesores que tienen las mismas creencias que ellos?

Desde nuestro punto de vista, no se puede intervenir sobre aquello que no se conoce, al igual que no es posible prevenir actitudes discriminatorias si uno mismo construye pensamientos discriminatorios y nadie le alerta de los mismos. Por ello, consideramos que la selección de centros de prácticas y de tutores académicos y profesionales es crucial, al igual que lo es el proporcionar una mayor importancia a la relación entre los centros de formación del profesorado y los centros de educación infantil y primaria, pues de esta manera la formación de los estudiantes será mucho más adaptada a la realidad de los centros educativos.

\section{PROPUESTA EDUCATIVA}

Como señalamos en el primer punto de este artículo, el proyecto "El futuro de la Educación" de la OCDE, "impulsa" de alguna manera el trabajo en las aulas de esta competencia mediante la inclusión de unidades de evaluación en la próxima edición del estudio PISA 2018. Para ayudar en este trabajo evaluativo y asegurar que los estudiantes, como futuros maestros, reciban cierta formación práctica antes de la llegada a los centros de prácticas, planteamos una propuesta basada en Cornejo y Pérez (2014) y Cornejo, Gómez-Jarabo y Jiménez (2017), desde la que se asume que la mayoría de los valores, actitudes y comportamientos se reflejan en la interacción comunicativa y son producto de la cultura que sesgan la percepción; por ello, resulta fundamental, y es donde radica el núcleo de la competencia global, entender cómo el conocimiento cultural puede proporcionar una comprensión de por qué las personas de culturas distintas perciben el mundo de una manera 
diferente a la propia. Como el mismo Hofstede (1980) señala, la cultura es simplemente la forma de hacer las cosas por aquí. Las cosas que hacemos independientemente de la cultura son las mismas, la forma de hacerlas o de abordarlas es lo que es distinto. El conocimiento aplicado a las diferencias a la hora de ver y de hacer las mismas cosas es lo que nos hace interculturalmente más competentes.

Recogemos la definición de competencia global de la OCDE, así como las cuatro premisas del modelo teórico de aprendizaje cultural de Paige (2005), que pone el acento en la interacción comunicativa como medio para adquirir conocimientos, habilidades y actitudes necesarias para desarrollar la competencia intercultural:

- Reconocimiento: ¿Cómo demuestra una persona que es competente para reconocer sus propios valores culturales y los de los otros?

- Respeto: ¿Cómo puede sopesar una persona distintas perspectivas de sus propios comportamientos culturales?

- Reconciliación: ¿Cuán competente es una persona para conciliar esas diferentes perspectivas?

- Realización: ¿Cómo se demuestra la competencia que posee una persona cuando se ejecutan acciones que concilien las diferencias culturales?

Nuestra propuesta se desarrolla a través de un modelo didáctico de dimensiones o aspectos culturales basadas en las investigaciones realizadas por Hofstede (1980), Trompenaars y HampdenTurner (1997) y Hall (1966) para el ámbito organizacional, pero que resultan de fundamental valor en el ámbito formativo del futuro maestro, puesto que cada dimensión define un aspecto cultural concreto, que proporciona una forma de acercamiento y de análisis a la cultura propia y a la del otro, y facilita el diseño de intervenciones que traduzcan valores como respeto y tolerancia, más trabajados en general desde la perspectiva teórica, en comportamientos concretos en los chavales. Las dimensiones culturales propuestas son: individualismo-colectivismo, manejo de la incertidumbre, universalismo-particularismo, estilo de comunicación, cortesía verbal, comunicación no verbal y masculinidad-feminidad. La metodología utilizada es el estudio de casos que presenta una interactuación problemática entre personas de diferentes culturas en relación con una dimensión cultural determinada, lo que sirve para que el grupo realice una primera aproximación y reconozca lo que sabe y lo que no sabe en relación con la causa que ha provocado la situación. Además, permite constatar cómo sus propias hipótesis están influidas por los parámetros culturales propios. A continuación, mostramos ejemplo de la dimensión alto-bajo contexto: 
A Ana Álvarez, coordinadora del proyecto Puente para la movilidad de estudiantes de secundaria del colegio Adalia le resultaba cada vez más díicil trabajar con el grupo del mismo proyecto en Japón. Hacía ya dos semanas que este grupo había llegado a Madr id para compartir objetivosy estrategias del proyecto, porque era la primer a vez que habia un acuerdo entre el colegio de Madrid con Tokio, pero empezaba a dudar que hubiera sido buena idea... eran amables y corteses $\sin$ duda pero a la hor a de intentar llegar a acuerdos en las reuniones, los colegas japoneses casi no decian nada. Eso s, siempre asentian y esbozaban una ligera sonrisa Ana ya habia intentado que cerr a an el acuerdo en la primera reunión, puesto que no habia previsto ninguna dificutad. Ana kía las cláusulas los japoneses sonreían, peor no parecian dispuestos a la firma y Ana no quería forzar la situación. En la segunda reunión, Ana se dio cuenta de que pasaba lo mismo.

Empezaba a ser una stuación estresante para ela. Empezó a pensar que no estaban de acuerdo con ago y no se ar evían a dec í b, por lo que les preguntó si tenían alguna duda sobre el acuerdo de colaboración. Ellos, callados sonreían, pero no decían nada Cuando se les preguntaba si estaban de acuerdo con sus sugerencias, ellos siempre decían "sí", pero no hacían nada que le indic ara a Ana que dieran seguimiento a las propuestas. Cuando terminó esta segunda reunión, era media mañana y Ana les invitó a tomar cớé en la sala de profesores. Se quedó muy sorprendida cuandovio que allísuscolegas preguntaban, hablaban y parecían contentosy anmados inter éándose por la vida del colegio, por los profesores y por ella misma Incluso le preguntaron si estaba casada y tenía hijos. Por útimo, al dirigirse de nuevo a la sala de reuniones ile propusier on ir a un Karaoke! por la noche! el gr upo de Japón, i parecía esperar que ella saliera con ellos regularmente!

\section{Formula una hipótesis}

1. ¿Qué crees que pasa aqui?

2. ¿Por qué piensas que el grupo del colegio de Japón se comporta asi, quizá son poco serios y quieren aprovechar la visita para divertirse?

3. ¿En tu opinión, Ana tiene razón en estar estresada?

4. ¿Se te ocurre alguna solución, qué harias tú si fueras Ana? Si no se te ocurre nada, lee el recuadro a continuación

1. Posteriormente, se presenta una sucinta fundamentación teórica respecto a la dimensión para verificar las hipótesis iniciales y, a partir de ahí, las actividades de interacción comunicativa con una progresión didáctica que va desde: 
2. Actividades de sensibilización y concienciación para descubrir realidades distintas. Todos nos enfrentamos a las mismas situaciones, pero con puntos de partida distintos. Surgen a partir del análisis del caso al inicio de cada unidad.

3. Actividades de relativización sobre qué es lo mejor o lo correcto en relación con la cada dimensión trabajada en la unidad, aplicando algunos parámetros culturales estudiados en la unidad.

4. Actividades de organización donde conceptualizamos los puntos clave aprendidos en la unidad con situaciones más complejas, que implican mayor autonomía. Son actividades de producción.

5. Actividad de implicación al final de cada unidad, donde se propone la creación y aplicación de todo lo aprendido en la unidad y que conlleva una reflexión para la autorregulación de su propio aprendizaje.

Se trata, así pues, de que el conocimiento, la comprensión y el pensamiento analítico y crítico que componen la competencia global se pongan en práctica simultáneamente. Son estos tres componentes los que definen la capacidad de los estudiantes para pensar interculturalmente (Bok, 2006) y los que serán evaluados en PISA.

Si bien la propuesta que se realiza en estas líneas va referida a la formación que deberían recibir los estudiantes antes de enfrentarse a sus prácticas y durante las mismas, también sería preciso reforzar los planes de estudios de las titulaciones de educación con asignaturas obligatorias en las que se aborden la interculturalidad y la comunicación intercultural, pues consideramos que, si bien la propuesta esbozada puede servir para sensibilizar a los estudiantes de cara a la realización del Practicum, no da respuesta a la falta de formación detectada en los Grados que habilitan para el ejercicio de maestro en este aspecto. Por ello, también es necesario mejorar la formación que los tutores de prácticas y los tutores universitarios tienen en relación con intercultura. Como se ha analizado en líneas anteriores, diversas investigaciones muestran que, por regla general, la formación que ha recibido la mayoría del profesorado es carente en ese sentido, sobre todo, cuando se formaron en una época en la que no existía la diversidad actual:

Si los futuros profesionales de la educación no están recibiendo hoy en día (cuando nuestra sociedad es tan diversa y plural) una formación inicial que les prepare para trabajar con estudiantes de diferentes nacionalidades, de diferente sexo, de diferente capacidad..., ¿Qué ocurre con los profesionales que se formaron cuando no existía tal pluralidad? Lógicamente, tampoco la recibieron. (Sánchez Delgado y Gómez-Jarabo, 2016, p. 177)

Por tanto, se hace preciso reforzar la formación de los tutores para que estén en condiciones de orientar a sus estudiantes adecuadamente en relación a esos aspectos. En concreto en la Comunidad de Madrid (Orden 2581/2016, p. 42) se indica: "La Consejería de Educación, Juventud y Deporte reconocerá la participación del profesorado tutor y coordinador de acuerdo con la normativa vigente, pudiendo organizar cursos de formación que serán reconocidos con 1 crédito de formación”. Podría aprovecharse esa posibilidad para formar en relación a los temas que venimos tratando en este capítulo, pues el profesorado difícilmente podrá formar y orientar sobre temas sobre los que está escasamente preparado. 


\section{CONCLUSIONES O CONSIDERACIONES FINALES}

Méndez Zaballos (2012) concluye en la necesidad de analizar la naturaleza específica del proceso de enseñanza y aprendizaje que tiene lugar en el Practicum y que dota a esta asignatura de entidad propia. En este sentido, en este artículo hemos ofrecido una propuesta de diseño para esta asignatura en relación con el desarrollo de la competencia global, basado en el análisis de necesidades de formación del futuro maestro respecto a esa competencia y que al tiempo sirve para llamar la atención sobre una posible renovación en el diseño conceptual del Practicum desde la universidad. No se trata de incluir cualquier tipo de formación, genérica, sino más bien respetar los siguientes aspectos:

- Incidir en las necesidades reales de los centros educativos.

- No limitar la formación a los contenidos conceptuales, apostando por una formación más práctica, menos memorística.

- Prestar especial atención a la dimensión afectiva.

- Reforzar el trabajo en equipo, el debate, la negociación y la reflexión compartida. (GómezJarabo \& Sánchez Delgado, 2017).

Por otra parte, dado que el Practicum proporciona a los estudiantes la posibilidad de acercarse a su futuro lugar de trabajo y de conocer la realidad del entorno educativo, reiteramos una vez más la necesidad de que ese acercamiento se produzca reforzando los sistemas de tutoría y supervisión, pues tal como indica Zabalza (2011, p. 33), "la experiencia vivida, por rica y estimulante que sea, puede quedar en nada si no va acompañada de una adecuada supervisión que oriente la reflexión”. Necesariamente, el refuerzo de los sistemas de supervisión y tutoría en el Practicum no solo pasa por incrementar las horas de atención al alumnado y la calidad de esa atención, sino que también requiere una mayor relación entre los tutores de las facultades y los de las instituciones educativas, pues de esa forma podrán proporcionarse respuestas educativas más adaptadas a la realidad y necesidades de cada estudiante y podrían detectarse más fácilmente los vacíos dejados por la formación recibida.

\section{REFERENCIAS BIBLIOGRÁFICAS}

Aguado Odina, M. T. (Coord.) (2006). Educación intercultural. Necesidades de formación del profesorado desde una perspectiva europea. Madrid: UNED.

Asamblea General de las Naciones Unidas (2016). Migración internacional y desarrollo. Recuperado de: http://www.un.org/esa/population/migration/hld/Text/Report $\% 20$ of $\% 20$ the $\% 20$ SG\%20(June\%2006)_Spanish.pdf(Fecha de consulta: 23/07/17).

Bennett, J. (1993). Towards ethno-relativism: a development model of intercultural sensivity. En R. Michael (Ed.), Education for Intercultural Experience (pp. 29-34). Yarmonth: Intercultural Press.

Bok, D. (2006). Our underachieving colleges: A candid look at how much students learn and why they should be learning more. Princeton: Princeton University Press. 
Byram, M., Gribkova, B., y Starkey, H. (2002). Developing the intercultural dimension in language teaching. A practical introduction for teachers. Estrasburgo: Consejo de Europa. Recuperado de: http://www.coe.int/t/dg4/linguistic/Source/Guide_dimintercult_EN.pdf (Fecha de consulta: 10/04/2016).

Calvo Buezas, T. (2003). La escuela ante la inmigración y el racismo. Orientaciones de educación intercultural. Madrid: Editorial Popular.

Cornejo, M. J., y Pérez L. (2014). El desarrollo de la competencia intercultural en educación formal. Estrategias para favorecer la integración social en primaria y secundaria. En F. J. Pozo y C. Peláez-Paz (Ed.), Educación social en situaciones de riesgo y conflicto en iberoamérica (pp. 259-267). Madrid: UCM.

Cornejo, M. J., Gómez-Jarabo, I. y Jiménez, E. (2017). Comunicación Intercultural. Desarrollo de habilidades en educación formal y no formal. Madrid: CCS.

Deardorff, D. K. (2006). Identification and assessment of intercultural competence as a student outcome of internationalization. Journal of Studies in International Education, 10(3), 241266. DOI: https://doi.org/10.1177/1028315306287002

Díaz-Aguado, M. J. (2002). Educación intercultural y aprendizaje cooperativo. Madrid: Pirámide.

Domínguez Garrido, M. C. (2006). Investigación y formación del profesorado en una sociedad intercultural. Madrid: Editorial Universitas.

García López, R. (2002). Addenda al XXI Seminario Interuniversitario de Teoría de la Educación: Globalización, Inmigración y Educación. XXI Seminario Interuniversitario de Teoría de la Educación: Globalización, Inmigración y Educación. Universidad de Granada. Recuperado de: www.ucm.es/info/site/docu/21 site/a2glopez.pdf (Fecha de consulta: 30/06/2017).

Gómez Barreto, I. M., Medina Revilla, A. y Gil Madrona, P. (2011). La competencia intercultural en el plan de estudios de Graduado en Maestro de Educación Infantil en la Universidad de Castilla La Mancha. ENSAYOS, Revista de la Facultad de Educación de Albacete, 26, 35-54. Recuperado de https://www.revista.uclm.es/index.php/ensayos/article/view/67

Gómez-Jarabo, I. (2015). Formación del profesorado para el tratamiento educativo de los conflictos sobre diversidad cultural y de género. (Tesis inédita de doctorado). Madrid (España): Universidad Complutense.

Gómez-Jarabo, I. y Sánchez Delgado, P. (2017). Formación del profesorado en cuestiones de género. [Teacher training on gender issues], en Revista de Comunicación de SEECI, 43, 53-68. DOI: http://dx.doi.org/10.15198/seeci2017.43.53-68

Hall, E. (1966). The Hidden Dimension. New York: Doubleday.

Hofstede, G. (1980) Culture's Consequences: International Differencences in Work-related Values. Berverly Hills: Sage.

Kazepides, T. (2012). Education as Dialogue. En T. Besley y M. P. Peters (Eds.), Interculturalism, Education and Dialogue (pp. 76-86). Nueva York: Peter Lang.

Leiva Olivencia, J. J. (2012). La formación en educación intercultural del profesorado y la comunidad educativa. REID, monográfico 2, 172-192. Recuperado de https://revistaselectronicas. ujaen.es/index.php/reid/article/view/1148/970

López-Reillo, P. (2006). Una oportunidad para aprender. La dimensión intercultural en la formación del profesorado. Tenerife: Cabildo de Tenerife. 
Méndez Zaballos, L. (2012). El conocimiento situado y los sistemas de actividad. Un modelo teórico para repensar el prácticum. Revista de Educación, 359, 629-642.

Mingote, C. y Requena, M. (Eds.) (2008). El malestar de los jóvenes. Contextos, raíces y experiencias. Madrid: Díaz de Santos.

OCDE (2016). Global competence for an inclusive world. Recuperado de: https://www.oecd.org/ education/Global-competency-for-an-inclusive-world.pdf (Fecha de consulta: 17/09/2017).

Orden ECI/3854/2007, de 27 de diciembre, por la que se establecen los requisitos para la verificación de los títulos universitarios oficiales que habiliten para el ejercicio de la profesión de Maestro en Educación Infantil. Boletín Oficial del Estado, Madrid, 29 diciembre, núm. 312, pp. 29781-29783. Recuperado de: https://www.boe.es/boe/dias/2007/12/29/pdfs/A5373553738.pdf

Orden ECI/3857/2007, de 27 de diciembre, por la que se establecen los requisitos para la verificación de los títulos universitarios oficiales que habiliten para el ejercicio de la profesión de Maestro en Educación Primaria. Boletín Oficial del Estado, Madrid, 29 diciembre 2007, núm. 312, pp. 29781-29783. Recuperado de: https://www.boe.es/boe/dias/2007/12/29/pdfs/ A53747-53750.pdf

Orden 2581/2016, de 17 de agosto, de la Consejería de Educación, Juventud y Deporte, por la que se establece el procedimiento para la realización del Practicum de los alumnos de los Grados de Magisterio, Pedagogía y Psicología, matriculados en Facultades y Escuelas Universitarias de Formación del Profesorado en centros de la Comunidad de Madrid durante el curso 20162017. Boletín Oficial de la Comunidad de Madrid, 31 de agosto, núm. 208. Recuperado de: www.madrid.org/wleg/servlet/Servidor?opcion=VerHtmlInfo\&idnorma=10629

Paige, M. (2005). Maximizar los estudios en el extranjero: mejora de las estrategias de la cultura y el aprendizaje de idiomas de nuestros estudiantes. $17^{a}$ Conferencia Anual de la Asociación Europea para la Educación Internacional, Cracovia, Polonia.

Palomero, J. E. (2006). Formación inicial de los profesionales de la educación en pedagogía intercultural: una asignatura pendiente. El caso de Aragón. Revista Interuniversitaria de Formación del Profesorado, 20(1), 207-230.

Ramos, G. y Schleicher, A. (2016). The Future of Education and Skills: an OCDE Education 2030 Framework. En OCDE (2016), Global competence for an inclusive world. Recuperado de: https://www.oecd.org/education/Global-competency-for-an-inclusive-world.pdf (Fecha de consulta: 17/09/2017).

Real Decreto 1393/2007, de 29 de octubre, de Ordenación de las enseñanzas universitarias oficiales. Boletín Oficial del Estado, Madrid, 30 de octubre, núm. 260, pp. 29781-29783. Recuperado de: https://www.boe.es/buscar/act.php?id=BOE-A-2007-18770

Rodríguez Izquierdo. R. M. (2008). Atención Educativa a la diversidad étnico-cultural: nuevas competencias profesionales del profesorado. Revista de currículum y formación del profesorado, 12(3), 1-12.

Rodríguez Marcos, A., González Aguado, P., Egea Reche, M. y Gutiérrez Ruiz, I. (2004). ¿Nos estamos preparando para construir la nueva sociedad intercultural? Patrones de creencias y actitudes de estudiantes que terminan Magisterio. En A. Medina, A. Rodríguez e A. Ibáñez (Coords.). Interculturalidad. Formación del profesorado y educación. Madrid: Pearson Edu- 
cation.

Sánchez Delgado, P. (2009). Formación del profesorado no universitario y Espacio Europeo de Educación Superior, en Revista de la Asociación de Inspectores de Educación de España, 10, 1-14. Recuperado de: https://avances.adide.org/index.php/ase/article/download/401/259 Sánchez Delgado, P. y Gómez-Jarabo, I. (2016). Estrategias de intervención desde la formación del profesorado y su acción en el aula. En J. L. Bernal Agudo (Coord.), XIV Congreso Interuniversitario De Organización De Instituciones Educativas (CIOIE). "Globalización y organizaciones educativas”. Libro de Simposios. Zaragoza: Universidad de Zaragoza.

Trompenaars, F. y Hampden-Turner, C. (1997). Riding the Waves of Culture. London: Nicholas Brealey.

Zabalza, M. A. (2011). El Practicum en la formación universitaria: estado de la cuestión. Revista de Educación, 354, 21-43. 